\title{
Incorporação de nanossílica em compósitos cimentícios
}

\section{Incorporation of nanosilica in cement composites}

\author{
Geovana Souza Ferreira Nogueira ${ }^{1}$, Nicole Schwantes-Cezario ${ }^{1}$, \\ Isabela Cristina Souza ${ }^{1}$, Caroline Dias Cavaleiro', \\ Maria Fernanda Porto ${ }^{1}$, Berenice Martins Toralles ${ }^{1}$
}

\footnotetext{
${ }^{1}$ Laboratório de Materiais de Construção Civil - CTU/UEL, Universidade Estadual de Londrina, Rodovia Celso Garcia Cid, km 380, Londrina, Paraná, Brasil. e-mail: geovanasouzanogueira@gmail.com; nicoleschwantes@hotmail.com; isabela07@gmail.com; caroldias.cdc95@gmail.com; mfeernanda.porto@gmail.com; betoralles@gmail.com
}

\begin{abstract}
RESUMO
A incorporação de nanossílica vem sendo estudada como uma alternativa para melhorar as características dos materiais cimentícios, pois tende a aumentar as reações pozolânicas e preencher os espaços intersticiais, diminuindo a porosidade e melhorando a resistência mecânica. Neste contexto, o presente estudo teve como objetivo avaliar o comportamento mecânico e as características microestruturais de três compósitos cimentícios: um de referência e dois contendo diferentes teores de nanossílica dispersas em policarboxilato (NDP), de 0,2 e $0,4 \%$, em relação à massa do cimento. O comportamento mecânico foi avaliado mediante o ensaio de resistência à compressão e resistência à tração por compressão diametral. Além disso, foram realizados ensaios de absorção de água e análise microestrutural em Microscópio Eletrônico de Varredura (MEV). A partir da análise dos resultados, verificou-se que a adição de NDP melhorou as propriedades mecânicas e as características microestruturais dos compósitos cimentícios. Sendo que o compósito contendo o maior teor de NDP $(0,4 \%)$ obteve melhores resultados aos 28 dias, apresentando um aumento de $55 \%$ na resistência à compressão, $79 \%$ na resistência à tração por compressão diametral e diminuindo em $40 \%$ a absorção de água, em relação à referência, possivelmente pela atuação da NDP. Porém, ao analisar a incorporação dos dois teores de NDP (0,2 e 0,4\%), se verificou que ao dobrar o teor não houve uma melhora significativa da resistência à compressão, pois a incorporação de $0,2 \%$ de NDP aumentou a resistência à compressão em $53 \%$ em relação à referência. Quanto à análise microestrutural em MEV, verificou-se um refinamento da microestrutura a partir da incorporação de NDP, mas não foram visualizadas diferenças entre os dois teores incorporados.
\end{abstract}

Palavras-chave: compósito cimentício, nanossílica, resistência mecânica.

\section{ABSTRACT}

The incorporation of the nanosilica has been studied as an alternative to improve the characteristics of cementitious materials because tends to increase the pozzolanic reactions and fill the interstitial spaces, decreasing the porosity and improving the compressive strength. In this context, the present study has the goal to evaluate the mechanical behavior and the microstructural characteristics of three cementitious composites: the reference e two with different levels of nanosilica dispersed in polycarboxylate (NDP), 0.2 and $0.4 \%$, in relation to the cement mass. The mechanical behavior was evaluated through the compressive strength test and tensile test (Brazilian denomination). Furthermore, were done water absorption tests and microstructural analysis by Scanning Electron Microscope (SEM). From the analysis of the results, it was verified that the addition of NDP improved the mechanical properties and the microstructural characteristics of cementitious composites. The NDP content $(0.4 \%)$ showed better results at 28 days, presenting a 55\% increase in compressive strength, $79 \%$ tensile test and a $40 \%$ decrease in the absorption of water, in relation to the reference, possibly through the NDPs performance. However, when analyzing the incorporation of the two NDP contents, it was verified that when the content was doubled there was no significant improvement in the compressive strength, since the incorporation of $0.2 \%$ of NDP increased the compressive strength in 53\% in relation to the reference. As for the microstructural analysis, a refinement of the structure was verified from the incorporation of NDP but no differences between the two incorporation was visualized. 
Keywords: cementitious composite, nanosilica, mechanical strength.

\section{INTRODUÇÃO}

Recentemente a incorporação de nanopartículas em compósitos cimentícios tem se apresentado como um campo de pesquisa promissor, pois tende a potencializar as propriedades físicas e mecânicas [1, 2, 3]. Compósitos são materiais de duas ou mais fases que buscam propriedades complementares a partir da combinação de diferentes elementos. Pesquisas na área de compósitos têm buscado combinar materiais com capacidade de melhorar as propriedades mecânicas e características microestruturais [4,5]. Concomitantemente a essas pesquisas, o desenvolvimento da nanotecnologia tem proporcionado grandes progressos na ciência e na engenharia dos materiais, apresentando um elevado potencial de melhoria e controle de suas propriedades [1, $3,4,8]$.

As nanopartículas apresentam maior reatividade quando comparadas às partículas de mesma composição química em escala maior, pois quanto menor é a partícula, maior é a superfície específica e consequentemente maior é a velocidade das reações químicas $[9,10,11,12]$. Nesse cenário, a nanossílica é uma das nanopartículas utilizadas em materiais cimentícios com o objetivo de melhorar as propriedades mecânicas e as características microestruturais, tornando-os mais resistentes e duráveis $[13,14,15,16,17,18]$.

A incorporação da nanossílica em materiais cimentícios possui principalmente duas formas de atuação: uma química, atuando como pozolana de alta reatividade, e outra física, atuando como fíler. Na atuação química, a nanossílica reage rapidamente com o hidróxido de cálcio $\left(\mathrm{Ca}(\mathrm{OH})_{2}\right)$ liberado durante a hidratação do cimento, formando compostos resistentes de silicato de cálcio hidratado $(\mathrm{C}-\mathrm{S}-\mathrm{H})$, que tendem a preencher os vazios capilares. $\mathrm{Na}$ atuação física, as nanopartículas atuam como filler, preenchendo os espaços intersticiais entre os grãos de cimento, resultando em um refinamento da microestrutura. Além disso, o efeito filler está ligado à formação de pontos de nucleação que contribuem para o aumento dos produtos de hidratação de cimento. Sendo assim, as duas formas de atuação contribuem para uma estrutura mais densa, menos porosa e, consequentemente, com menor permeabilidade e maior resistência $[4,6,10]$.

Devido à alta reatividade, as nanopartículas de sílica podem formar aglomerações que tendem a prejudicar o preenchimento dos espaços intersticiais, pois os grumos podem afastar as partículas de cimento, acarretando em um aumento dos vazios. Essas aglomerações também podem reduzir as reações pozolânicas, por diminuírem a superfície específica. Além disso, a formação aglomerações maiores pode ocasionar em um aumento na absorção de água livre da mistura, comprometendo a fluidez do sistema [19,20].

A Figura 1 ilustra as aglomerações de nanossílica, em que a Figura 1-(a), demonstra as nanopartículas preenchendo os espaços intersticiais entre as partículas de cimento. Já a Figura 1-(b) mostra a formação de pequenos grumos de nanossílica que prejudicam o preenchimento dos espaços vazios, tendendo a afastar as partículas a sua volta, aumentando os espaços vazios e consumindo um pouco da água livre que contribuiria para a melhoria da fluidez. Figura 1-(c) apresenta a formação de grumos maiores, que absorvem mais água livre, comprometendo a fluidez do sistema [19].

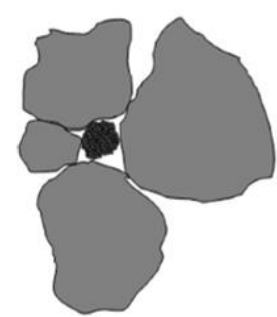

(a)

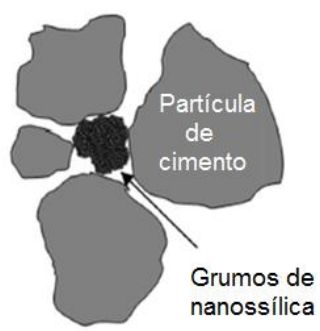

(b)

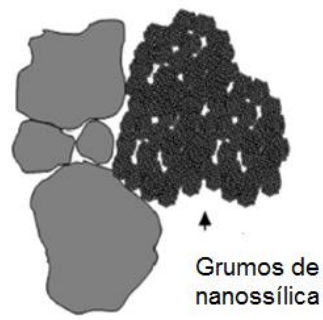

(c)

Figura 1: Ilustração das aglomerações de nanossílica. Fonte: Adaptado KONG et al. [19].

Cabe ressaltar que quanto maior a superfície específica da nanossílica maior será o consumo de água nas reações de hidratação, podendo prejudicar a fluidez do compósito caso a quantidade de água não seja ajustada para o teor incorporado. Para minimizar esses problemas, pode-se utilizar a nanossílica dispersa em aditivo superplastificante a base de policarboxilato, a fim de obter uma maior dispersão das nanopartículas e melhorar a ação dos mecanismos químicos e físicos, não sendo necessário adicionar água para manter a fluidez do sistema. 
MALLMAN [21] e CASTRO E SOUZA [22] estudaram a adição teores entre 0,5 a 4\% de nanossílica dispersa em policarboxilato (NDP) em relação à massa de cimento e verificaram que houve uma diminuição da resistência à compressão em relação à referência. Em contrapartida, NOLLI FILHO [23] estudou incorporação de $0,39 \%$ de NDP e obteve um aumento na resistência à compressão em torno de $12 \%$.

Com base no exposto, o presente trabalho objetivou verificar a influência da incorporação de 0,2 e 0,4\% de nanossílica dispersa em policarboxilato (NDP) em compósitos cimentícios, mediante a avaliação do comportamento mecânico e características microestruturais.

\section{MATERIAIS E MÉTODOS}

Este trabalho estudou de forma comparativa três compósitos cimentícios: um compósito de referência e dois compósitos com incorporação de diferentes teores de nanossílica dispersa em policarboxilato (NDP).

\subsection{Materiais}

Os materiais utilizados para a realização dos experimentos foram:

- $\quad$ Cimento Portland CP V-ARI, por não conter adições pozolânicas ou escórias;

- $\quad$ Nanossílica dispersa em policarboxilato (NDP);

- $\quad$ Areia de rio (média e fina de quartzo);

- Água potável.

A Tabela 1 apresenta as especificações do cimento CPV ARI, de acordo com informações repassadas pelo fabricante, e as exigências da NBR 05733/1991 [24].

Tabela 1: Características do cimento CPV ARI Estrutura da Cauê e exigências da NBR 05733/1991.

\begin{tabular}{c|c|c|c|c|c}
\hline \multirow{2}{*}{ IDADES } & \multicolumn{4}{|c|}{ RESISTÊNCIA À COMPRESSÃO (MPa) } & \multirow{2}{*}{$\begin{array}{c}\text { BLAINE } \\
\text { (cm } / \mathbf{g})\end{array}$} \\
\cline { 2 - 6 } & 24 HORAS & 3 DIAS & 7 DIAS & 28 DIAS & $\geq 3000$ \\
\hline NBR 05733/1991 & $\geq 14$ & $\geq 24$ & $\geq 34$ & - & 5330 \\
\hline CPV ARI Cauê & 27 & 37 & 42 & 48 & 5 \\
\hline
\end{tabular}

Fonte: Empresa Cauê e NBR 05733/1991.

\subsection{Método}

Os componentes utilizados para confecção dos compósitos cimentícios estudados estão apresentados na Tabela 2, assim como as nomenclaturas adotadas. Cabe destacar que os teores fixados de NDP foram escolhidos a partir da análise dos resultados obtidos nos estudos de MALLMAN [21], CASTRO E SOUZA [22] e NOLLI FILHO [23].

Tabela 2: Nomenclatura dos Compósitos Cimentícios (CC).

\begin{tabular}{l|l|l}
\hline COMPÓSITO & \multicolumn{1}{|c|}{ COMPONENTES } & NOMENCLATURA \\
\hline I & Referência (cimento + areia + água); & CC I $(0)$ \\
\hline II & Referência + 0,2\% NDP em relação à massa de cimento & CC II $(0,2)$ \\
\hline III & Referência + 0,4\% NDP em relação à massa de cimento & CC III $(0,4)$ \\
\hline
\end{tabular}

Fonte: Autores.

Com a finalidade de escolher a melhor distribuição granulométrica do agregado miúdo foram analisadas diversas combinações de duas areias com granulometrias diferentes, a fim de escolher a composição granulométrica com a maior massa unitária e o menor índice de vazios. Na sequência, para determinar as características da composição escolhida, foram realizados os ensaios descritos na Tabela 3, seguindo as instruções das referidas normas. 
Tabela 3: Ensaios realizados para a caracterização da areia.

\begin{tabular}{l|l}
\hline \multicolumn{1}{c|}{ ENSAIOS } & \multicolumn{1}{c}{ NORMAS } \\
\hline Composição granulométrica & NBR NM 248/2003 [25] \\
\hline Massa específica & NBR NM 52/2009 [26] \\
\hline Massa unitária & NBR NM 45/2006 [27] \\
\hline Absorção de água & NBR NM 30/2001 [28] \\
\hline
\end{tabular}

Fonte: Autores.

Foram testadas três tipos de nanossílicas dispersas em policarboxilato (A, B e C) com o intuito de selecionar uma NDP com o maior teor de silício, suas especificações estão apresentadas na Tabela 4.

Tabela 4: Especificações das NDP's, conforme repassado pelo fabricante.

\begin{tabular}{l|c|c|c}
\hline \multicolumn{1}{c|}{ PROPRIEDADES } & NDP A & NDP B & NDP C \\
\hline Densidade relativa & 1,07 & 1,09 & 1,06 \\
\hline Teor de sólidos convencional & 35,56 & 48,5 & 30 \\
\hline Valores de pH & 2,9 & 2,99 & 2,9 \\
\hline Teor de cloreto & $<0,1 \%$ & $<0,1 \%$ & $<0,1 \%$ \\
\hline Teor de álcalis & - & - & - \\
\hline
\end{tabular}

Fonte: Fabricante.

Desta maneira, para selecionar a NDP com o maior teor de nanossílica foi realizado o ensaio de fluorescência de raios $\mathrm{X}$ com o equipamento de espectrometria de energia dispersiva de raios-X (EDX). Como este equipamento não analisa amostras líquidas, foi necessário liofilizar as amostras. O processo de liofilização foi baseado na ASTM E1342 [29], em que as amostras são desidratadas por sublimação, levando-as congeladas em câmaras herméticas. Nesse processo, todo o ar de dentro das câmeras herméticas foi removido através de bombas de alto vácuo, criando a condição para que ocorra a sublimação da água.

Na sequência, os compósitos cimentícios foram elaborados e realizou-se ensaios no estado fresco e endurecido de acordo com a normatização nacional. Os ensaios realizados estão apresentados nas Tabela 5 e 6.

Tabela 5: Ensaios realizados no estado fresco e suas respectivas normas.

Fonte: Autores.

\begin{tabular}{l|l}
\hline \multicolumn{1}{c|}{ ENSAIOS } & \multicolumn{1}{c}{ NORMAS } \\
\hline Consistência & NBR NM 67/1998 [30] \\
\hline Densidade de massa & NBR 13278/2005 [31] \\
\hline
\end{tabular}

Tabela 6: Ensaios realizados no estado endurecido e suas respectivas normas.

\begin{tabular}{l|c}
\hline \multicolumn{1}{c|}{ ENSAIOS } & NORMAS \\
\hline Resistência à compressão - Prensa Hidráulica PCE 200 PLUS & NBR 7215/1996 [32] \\
\hline $\begin{array}{l}\text { Resistência à tração por compressão diametral - Prensa Hidráulica PCE 200 } \\
\text { PLUS }\end{array}$ & NBR 7222/2011[33] \\
\hline Absorção por imersão & NBR 9778/2009 [34] \\
\hline Análise microestrutural no MEV modelo FEI Quanta 200 & - \\
\hline
\end{tabular}

Fonte: Autores

Para a realização dos ensaios de resistência mecânica foram moldados 8 corpos de prova cilíndricos de dimensões 50x100 mm, sendo 4 analisados aos 7 dias de idade e os outros 4 aos 28 dias. O equipamento utilizado para ambos os ensaios de resistência mecânica foi a Prensa Hidráulica de acionamento elétrico, mo- 
delo PCE 200 PLUS, com capacidade de 200.000 kgf da marca EMIC. Os parâmetros de ensaio seguiram a normatização da Tabela 6.

O traço adotado para elaboração dos compósitos foi de 1:1 (cimento: areia), em massa, pois buscavase um compósito de elevada resistência. Fixou-se a consistência em $260 \mathrm{~mm} \pm 10 \mathrm{~mm}$, dessa forma a relação água/cimento (a/c) variou, pois a água foi adicionada até se obter a consistência desejada. Na Tabela 7 estão apresentadas as quantidades em massa dos componentes utilizados nos compósitos estudados.

Tabela 7: Consumo dos materiais constituintes dos compósitos cimentícios.

\begin{tabular}{c|c|c|c}
\hline \multirow{2}{*}{ COMPÓSITOS } & \multicolumn{3}{|c}{ CONSUMO DE MATERIAIS } \\
\cline { 2 - 4 } & CIMENTO $(\mathbf{g})$ & AREIA $(\mathbf{g})$ & NDP $(\mathbf{m L})^{\star}$ \\
\hline I & 3300 & 3300 & - \\
\hline II & 3300 & 3300 & 6,06 \\
\hline III & 3300 & 3300 & 12,11 \\
\hline
\end{tabular}

* Em relação a massa de cimento.

Fonte: Autores.

\section{RESULTADOS E DISCUSSÕES}

\subsection{Caracterização dos Materiais}

A fim de escolher a composição com o menor índice de vazios e otimizar a distribuição granulométrica, foram realizados ensaios de massa unitária compactada de diversas combinações de areia média e areia fina. Os resultados obtidos estão demostrados na Figura 2.

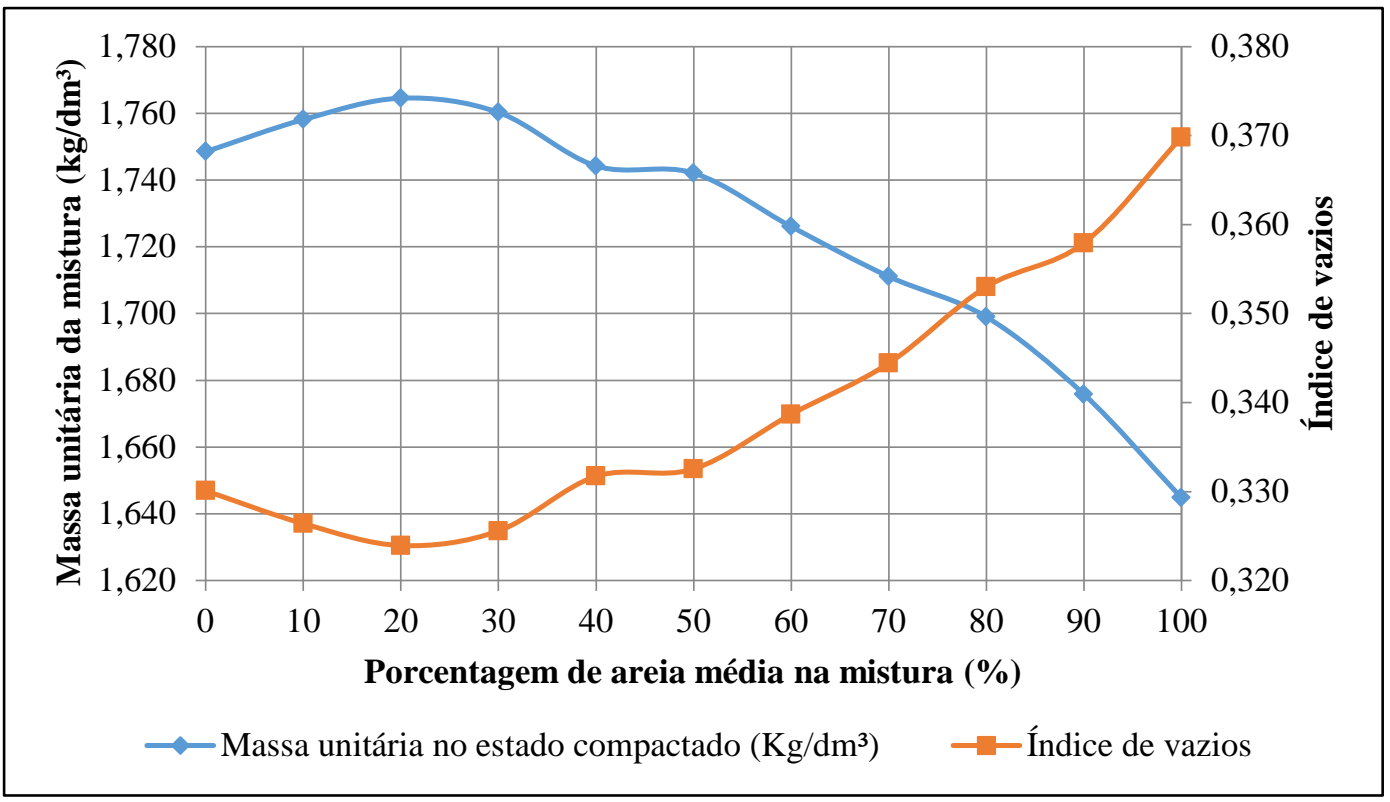

Figura 2: Massa unitária e índice de vazios das composições de areia. Fonte: Autores.

A partir da análise da Figura 2, verificou-se que a composição com a maior massa unitária e o menor índice de vazios foi de aproximadamente $20 \%$ de areia fina e $80 \%$ de areia média, sendo essa a composição final utilizada para a confecção dos compósitos cimentícios. Na sequência, a Figura 3 ilustra a curva granulométrica da composição escolhida, em que é possível visualizar que a curva granulométrica da combinação se encontra dentro dos limites utilizáveis estabelecidos por norma e apresentou uma granulometria contínua. 


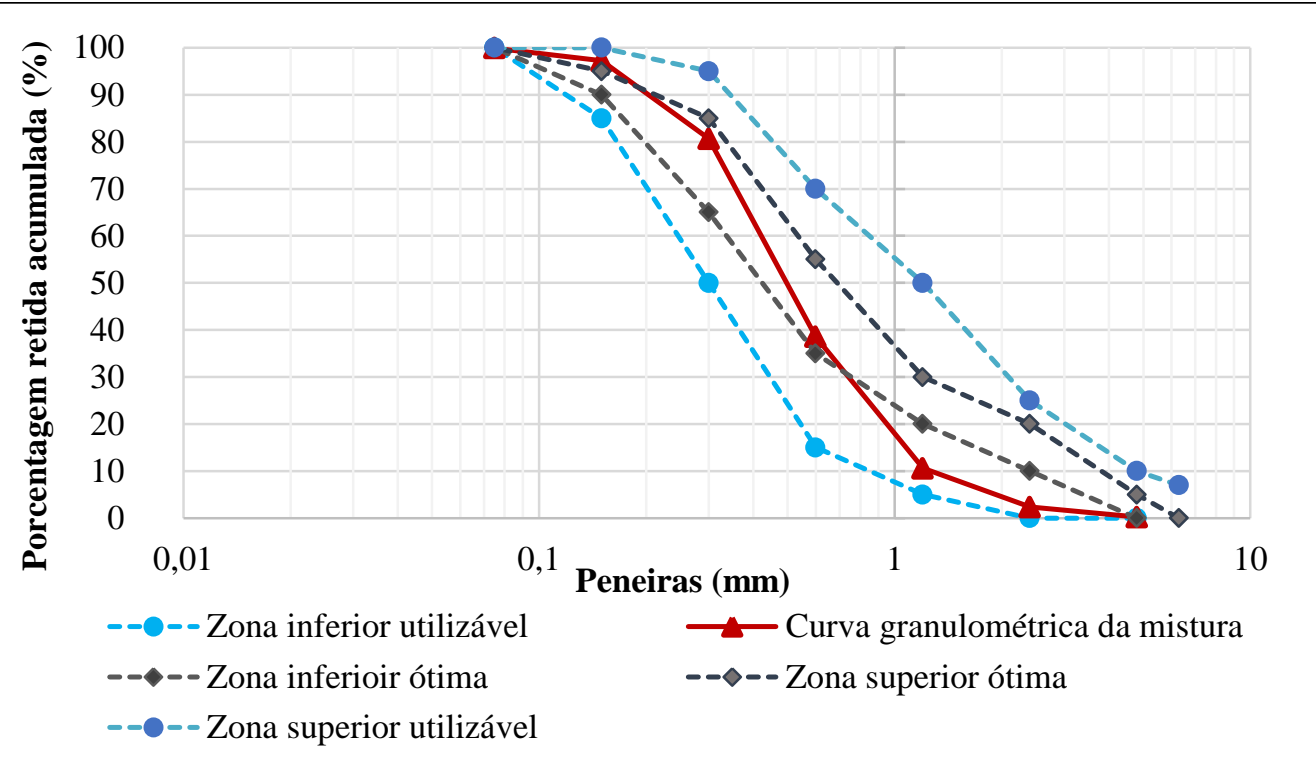

Figura 3: Curva granulométrica da composição escolhida. Fonte: Autores.

Além da composição granulométrica foram realizados ensaios de massa específica, absorção de água, massa unitária e módulo de finura, cujos resultados podem ser visualizados na Tabela 8.

Tabela 8: Resultados de massa específica, absorção de água, massa unitária e módulo de finura da combinação.

Fonte: Autores.

\begin{tabular}{l|c}
\hline \multicolumn{1}{c|}{ ENSAIOS } & RESULTADOS \\
\hline Massa específica & $2,61 \mathrm{~g} / \mathrm{cm}^{3}$ \\
\hline Absorção de água & $0,57 \%$ \\
\hline Massa unitária & $1,765 \mathrm{~g} / \mathrm{cm}^{3}$ \\
\hline Módulo de finura & 2,29 \\
\hline
\end{tabular}

Na sequência as NDP's utilizadas foram ensaiadas por espectrometria de energia dispersiva de raios-X (EDX), a fim de verificar a presença de nanossílica nas amostras, a partir da quantidade de silício detectada. Além do silício, o método detectou a presença de diversos elementos químicos, como pode ser observado na Tabela 9.

Tabela 9: Elementos químicos detectados nas amostras de NDP's estudadas.

\begin{tabular}{l|c|c|c}
\hline \multirow{2}{*}{ ELEMENTO QUímICo } & \multicolumn{3}{|c}{ QUANTIDADE (\%) } \\
\cline { 2 - 4 } & $\mathbf{A}$ & $\mathbf{B}$ & $\mathbf{C}$ \\
\hline Carbono & 58,22 & 60,19 & 60,30 \\
\hline Oxigênio & 40,15 & 39,04 & 39,16 \\
\hline Sódio & 1,13 & 0,38 & 0,29 \\
\hline Silício & $\mathbf{0 , 1 3}$ & $\mathbf{0 , 1 6}$ & $\mathbf{0 , 0 9}$ \\
\hline Enxofre & 0,36 & 0,24 & 0,13 \\
\hline Alumínio & - & - & 0,02 \\
\hline Total & 100 & 100 & 100 \\
\hline
\end{tabular}

Fonte: Autores.

A partir da análise da Tabela 9, verificou-se que dentre as três amostras ensaiados, a NDP B foi a que apresentou a maior quantidade de silício no ensaio de EDX e por este móvito esta NDP foi selecionada para a 
aplicação nos compósitos.

\subsection{Propriedades dos compósitos cimentícios}

\subsubsection{Propriedades no estado fresco}

$\mathrm{Na}$ Tabela 10 estão apresentadas as relações a/c referentes a cada compósito, para obter a consistência de 260+/- 10mm, bem como os valores do índice de consistência, relação água/materiais secos, densidade de massa e densidade de massa sem vazios.

Tabela 10: Relações e propriedades dos compósitos cimentícios no estado fresco.

\begin{tabular}{l|c|c|c|c|c}
\hline compósITOS & $\begin{array}{c}\text { RELAÇÃO } \\
\text { ÁGUA/CIMENTO } \\
(\mathbf{a} / \mathbf{c})\end{array}$ & $\begin{array}{c}\text { RELAÇÃO } \\
\text { ÁGUA/MATERIAIS } \\
\text { SECOS }\end{array}$ & $\begin{array}{c}\text { ÍNDICE DE } \\
\text { CONSISTÊNCIA } \\
(\mathbf{m m})\end{array}$ & $\begin{array}{c}\text { DENSIDADE } \\
\text { DE MASSA } \\
\left(\mathbf{g} / \mathbf{c m}^{\mathbf{3}}\right)\end{array}$ & $\begin{array}{c}\text { DENSIDADE DE MAS- } \\
\text { SA SEM VAZIOS } \\
\left(\mathbf{g} / \mathbf{c m}^{\mathbf{3}}\right)\end{array}$ \\
\hline CC I $(0)$ & 0,38 & 0,19 & 266 & 2,20 & 2,20 \\
\hline CC II $(0,2)$ & 0,30 & 0,15 & 261 & 2,24 & 2,29 \\
\hline CC III $(0,4)$ & 0,27 & 0,13 & 265 & 2,28 & 2,33 \\
\hline
\end{tabular}

Fonte: Autores.

O compósito III foi o que apresentou a menor relação a/c e consequentemente possui a maior densidade de massa. Acredita-se que tal resultado se justifique pela atuação da incorporação de 0,4\% da NDP.

Quanto à relação água/materiais secos verificou-se que para manter a consistência de $260 \mathrm{~mm} \pm 10$ mm, foi necessária uma relação de 0,19 no compósito I, enquanto que para o compósito III a relação foi de 0,13 . Sendo que o compósito contendo o maior teor de NDP demandou menos água que os demais pelo fato da nanossílica estar dispersa em policarboxilato.

\subsubsection{Propriedades no estado endurecido}

\subsubsection{Resistência à compressão}

Os resultados obtidos no ensaio de resistência à compressão foram graficados e estão apresentados na Figura 4.

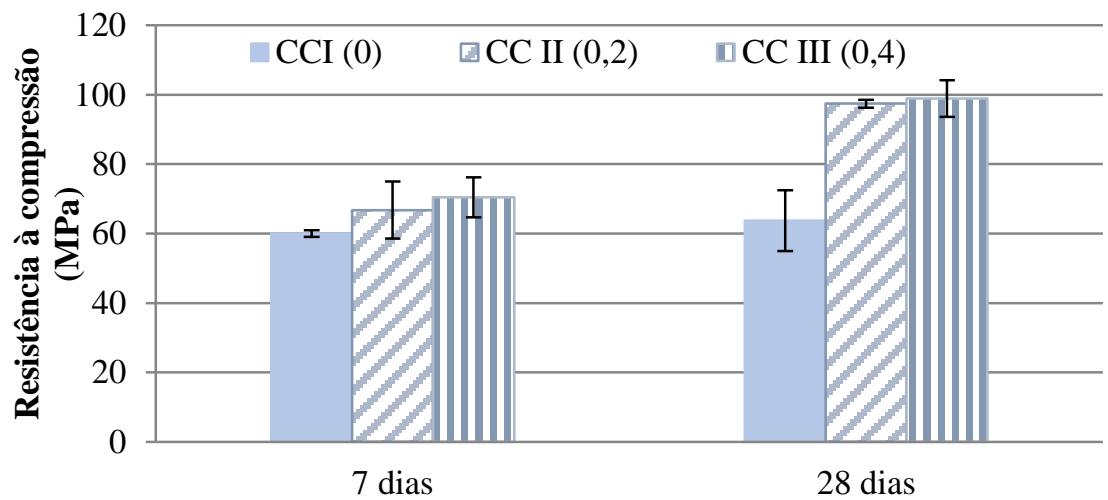

Figura 4: Resistência à compressão dos compósitos I, II, III. Fonte: Autores.

Aos 7 dias de cura, a resistência à compressão aumentou com o acréscimo do teor de NDP, sendo esse aumento de $11,2 \%$ e $17 \%$, em relação à referência, para os teores de $0,2 \%$ e $0,4 \%$ respectivamente. Já aos 28 dias esses aumentos foram de 52,8\% e 55\%. Analisando os teores de NDP utilizados, observou-se que o aumento da resistência à compressão não foi proporcional ao aumento do teor de NDP. A mesma tendência foi observada no trabalho realizado por SHIH et al. [6], no qual o fato de dobrar o teor de nanossílica de 0,2 a $0,4 \%$ não influenciou no aumento de resistência na mesma proporção. Porém esses autores não utilizaram policarboxilato nas misturas das pastas de cimento estudadas. 
É interessante mencionar que a incorporação de teores menores que 0,4\% de NDP apresentaram melhorias na resistência à compressão, assim como observado no trabalho de NOLLI FILHO [23], enquanto que teores maiores que $1 \%$ não resultaram em ganhos de resistência, conforme o resultado de MALLMAN [21] e CASTRO E SOUZA [22].

\subsubsection{Resistência à tração por compressão diametral}

Na Figura 5 pode-se visualizar o gráfico de resistência à tração por compressão diametral dos compósitos I, II e III.

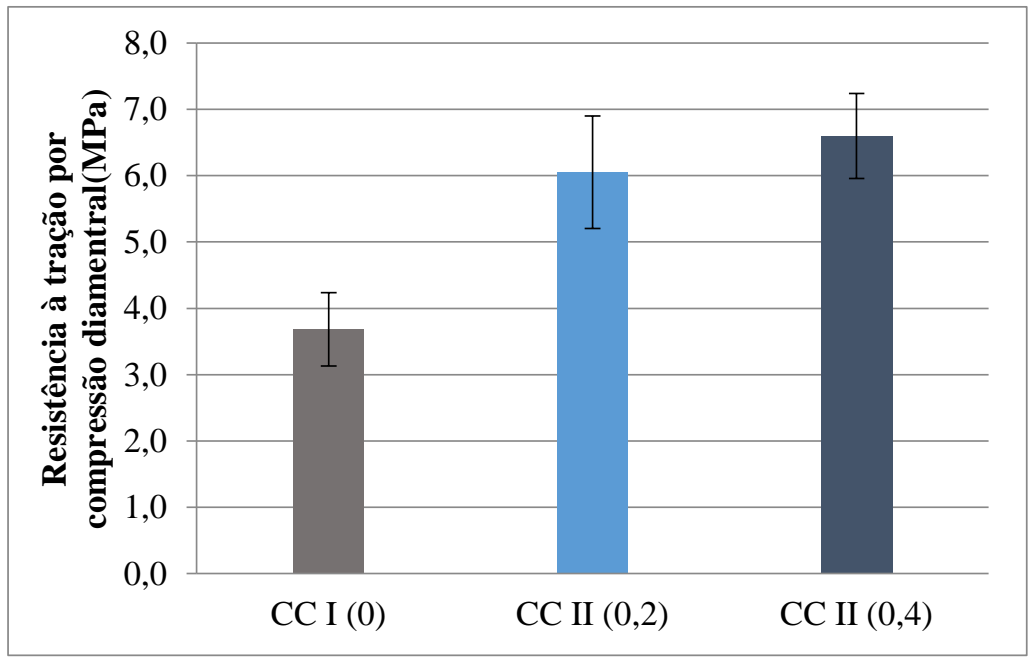

Figura 5: Resistência à tração por compressão diametral dos compósitos I, II, III. Fonte: Autores.

Aos 28 dias de idade, a resistência à tração por compressão diametral aumentou com o acréscimo do teor de NDP, sendo esse aumento de $64 \%$ e $79 \%$, em relação à referência, para os teores de $0,2 \%$ e $0,4 \%$ respectivamente.

\subsubsection{Absorção por imersão}

Na Figura 6 visualiza-se os resultados de absorção após saturação e fervura dos compósitos I, II e III.

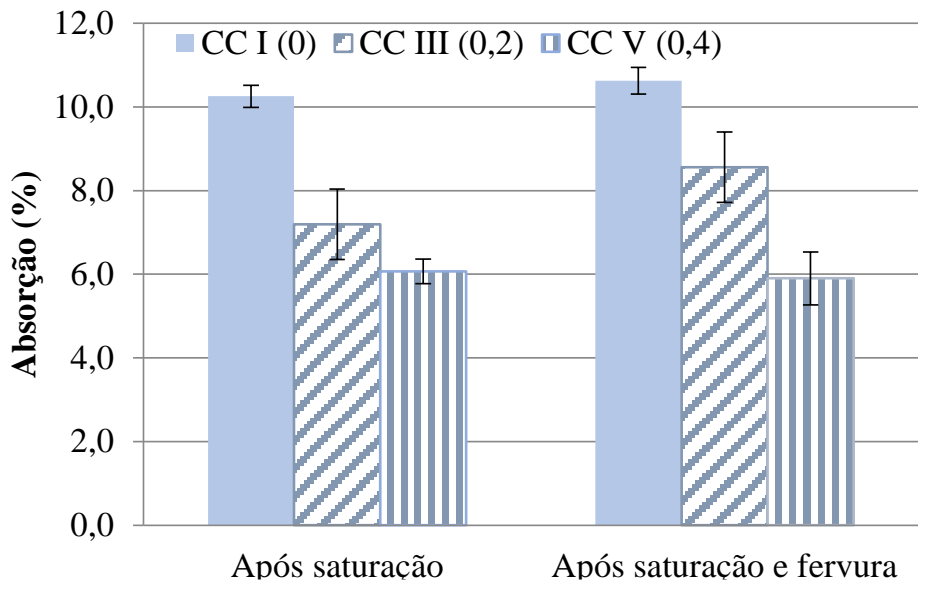

Figura 6: Absorção de água dos compósitos I, II, III. Fonte: Autores.

Pode-se notar que a adição de NDP em 0,2 e 0,4\% diminuíram a absorção após saturação em $30 \%$ e $40 \%$, em relação à referência e, também reduziram a absorção após saturação e fervura em $20 \%$ e $45 \%$.

O compósito III, com a menor relação a/c e a maior densidade de massa, apresentou a menor absorção, tanto após a imersão quanto após a fervura, quando comparado aos demais compósitos. Acredita-se que tal fato ocorreu devido ao refinamento da microestrutura, ocasionado pela adição de nanossílica dispersa em policarboxilato. 


\subsubsection{Análise microestrutural no MEV}

Por fim, foi realizada análise em Microscópio Eletrônico de Varredura (MEV) no Laboratório de Microscopia Eletrônica e Micro Análise - LMEN, da Universidade Estadual de Londrina - UEL, no Espectrofotômetro modelo FEI Quanta 200. O feixe de elétrons principal foi provocado por um filamento de tungstênio cuja tensão e corrente de operação foi de $20 \mathrm{kV}$ e $94 \mu \mathrm{A}$, respectivamente. Nas Figuras 7 e 8 estão apresentada todas as micrografias realizadas.

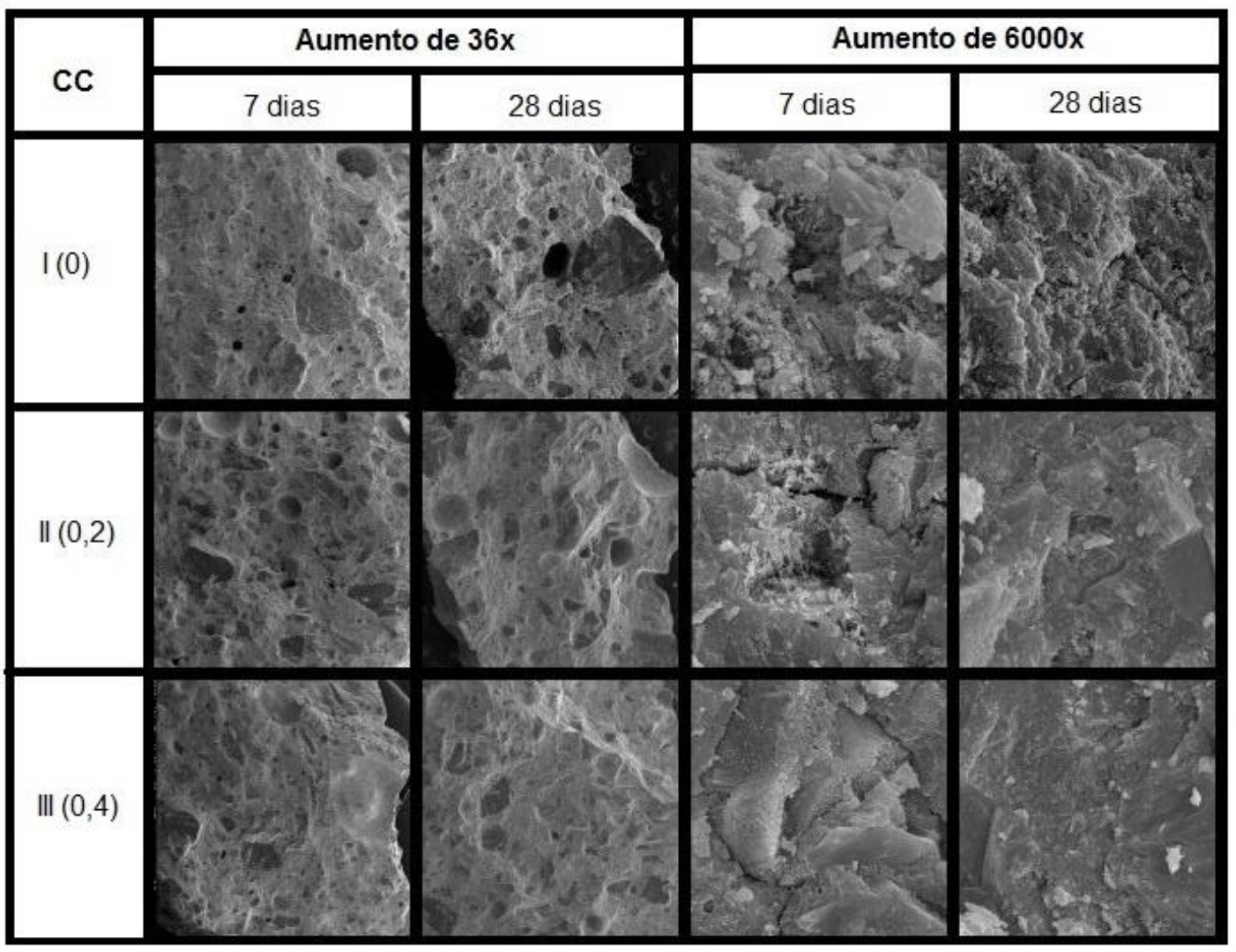

Figura 7: Comparativo das micrografias dos compósitos cimentícios. Fonte: Autores.

Perante a análise da Figura 7 pode-se observar que houve uma melhora na densidade das amostras de acordo com que sua microestrutura era refinada a partir da adição de NDP, sendo que visualmente os compósitos II e III não apresentaram diferenças na microestrutura, considerando que foi dobrada a quantidade de NDP. Na sequência, pode-se visualizar na Figura 8 os produtos de hidratação do cimento, como silicato de cálcio hidratado $(\mathrm{C}-\mathrm{S}-\mathrm{H})$ e cristais de etringita, representados na figura por Etr. 


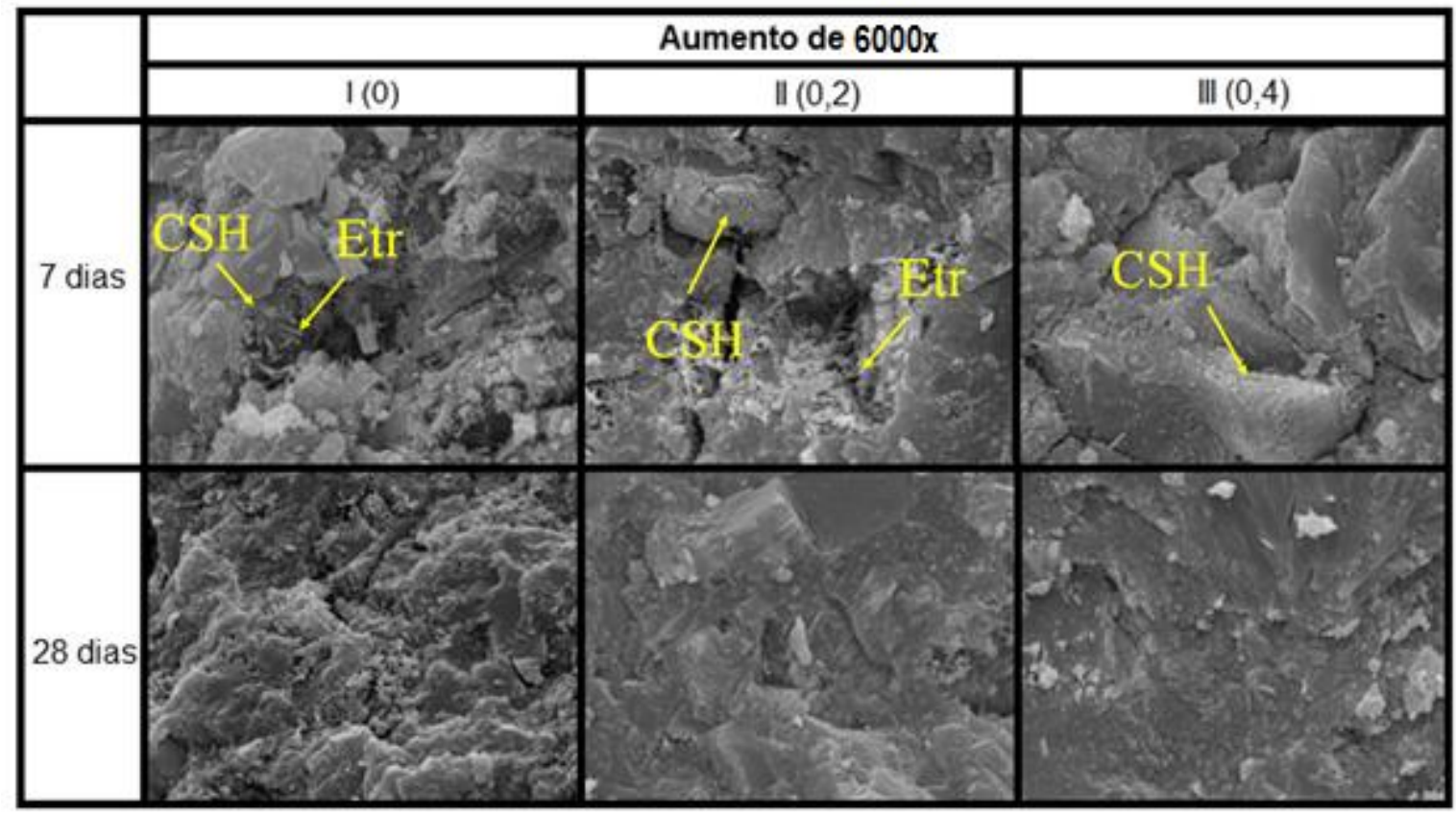

Figura 8: Comparativo das micrografias dos compósitos cimentícios com indicação dos produtos de hidratação do cimento. Fonte: Autores.

A partir da análise das imagens da Figura 8 verificou-se a presença de silicato de cálcio hidratado (CSH) em todas as amostras. Os cristais de etringita (Etr) foram visualizados apenas nos compósitos CC I e CC II, sendo que no compósito com $0,4 \%$ de NDP, não foi possível verificar a presença destes cristais.

\section{CONCLUSÕES}

Em uma análise global dos resultados verificou-se que todos os compósitos contendo incorporações de NDP apresentaram melhorias na resistência mecânica e nas características microestruturais, quando comparado com o compósito de referência, possivelmente pela atuação da NDP na formação de silicato de cálcio hidratado (C-S-H) e pelo refinamento da microestrutura devido ao preenchimento de espaços intersticiais. Vale ressaltar que os teores de NDP estudados acarretaram nessas melhorias, pois outros pesquisadores [21,22] que incorporaram teores maiores que os estudados não obtiveram melhorias nos resultados.

Além disso, verificou-se que a maior resistência mecânica foi alcançada ao incorporar 0,4\% de NDP. Porém, quando o aumento do teor de NDP (de 0,2 para $0,4 \%$ ) foi avaliado, as diferenças nas propriedades estudadas não foram significativas, considerando que o teor de NDP foi dobrado. Sendo que é importante salientar que esta pesquisa avaliou apenas a nanossílica dispersa em aditivo a base de policarboxilato, o que não possibilitou a verificação isolada do efeito das nanopartículas de sílica.

\section{AGRADECIMENTOS}

À Universidade Estadual de Londrina e aos técnicos do laboratório de materiais de construção civil e de microscopia eletrônica de varredura e microanálise.

\section{BIBLIOGRAFIA}

[1] XU, J., WANG, B., ZUO, J., "Modification effects of nanosilica on the interfacial transition zone in concrete: A multiscale approach", Cement and Concrete Composites, v. 81, p. 1-10, Ag. 2017.

[2] GAITERO, J. J., CAMPILlO, I., GUERRERO, A., "Reduction of the calcium leaching rate of cement paste by addition of silica nanoparticles", Cement and Concrete Research, v. 38, n. 8, pp. 1112-1118, 2008.

[3] JAIN, J. A., NEITHALATH, N, "Beneficial effects of small amount of nano-silica on the chemical stability of cement pastes exposed to neutral pH environments", ACI Fall convention, Dez. 2009.

[4] CALLISTER JUNIOR, W.D., Ciência e Engenharia de Materiais: Uma Introdução, 5 ed., Rio de Janeiro, LTC- Livros Técnicos e Científicos Editora S.A., 1999. 
[5] HENCHE, D., Composto cimentício de alta resistência com adição de nanotubo de carbono, Dissertação de M.Sc., Universidade Federal do Paraná, Curitiba, PR, Brasil, 2013.

[6] GLEIZE, P.J.P., Nanotecnologia e materiais de construção, In: ISAIA, G. C, "Materiais de construção civil e princípios de ciência e engenharia de materiais”, cap 50, 1 ed, v. 2, São Paulo, Editora Ibracon, 2007.

[7] TOBÓN, J.I., BAENA, O.J.R., BERNABEU, J.J.P., “Adición de nanopartículas al cemento Portland”, Dyna, v.74, n. 152, pp. 277-291, Jul. 2007.

[8] SANCHEZ, F., SOBOLEV, K. "Nanotechnology in concrete-a review", Construction and building materials, v. 24, n. 11, pp. 2060-2071, Mai. 2010.

[9] JO, B.W., KIM, C.H., TAE, G.H., et al., "Characteristics of cement mortar with nano-SiO2 particles”, Construction and building materials, v. 21, n. 6, pp. 1351-1355, Jun 2007.

[10] SHIH, J.Y., CHANG, T.P., HSIO, T.C., "Effect of nanosilica on characterization of Portland cement composite”, Materials Science and Engineering, v. 424, pp. 266-274, Mar 2006.

[11] BERGNA, H. E., ROBERTS, William O., Colloidal silica: fundamentals and applications, v.131, Flórida, Editora CRC Press, 2005.

[12] R. K, ILER. "The chemistry of silica: solubility, polymerization, colloid and surface properties and biochemistry”, John Wiley \&Sons Inc, 1979.

[13] DANTAS, M.H.D.O., Avaliação dos efeitos da nano sílica em pó e coloidal em pasta de cimento Portlland, Dissertação de M.Sc., Universidade Federal de Pernambuco, Caruaru, PE, Brasil, 2013.

[14] KONTOLEONTOS, F., TSAKIRIDIS, P.E., MARINOS, A., et al., "Influence of colloidal nanossílica on ultrafine cemente hydration: Physicochemical and microstructural characterization", Construction and building materials, v. 35, pp. 347-360, Mai 2012.

[15] SENFF, L., LABRINCHA J.A., FERRREIRA V.M., et al., "Effect of nano-silica on rheology and fresh properties of cement pastes and mortars", Construction and Building Materials, v. 23, pp. 2487-2491, 2009.

[16] BIRICIK, H., SARIER, N., "Comparative study of the characteristics of nano silica, silica fume and fly ash incorporated cement mortars", Materials Research, v. 17, n.3, pp. 570-582, 2014.

[17] LTIFI, M., GUEFRECH, A., MOUNANGA, P., et al., "Experimental study of the effect of addition of nano-silica on the behavior of cement mortars, Procedia Engineering, v. 10, n. pp. 900-905, 2011.

[18] NILI, M., EHSANI, A., SHABANI, K., "Influence of Nano-SiO2 and Microsilica on concrete performance", In: International Conference on Sustainable Construction Materials and Technologies, pp. 1-5, Ancona, Jun. 2010.

[19] KONG, D., SU, Y., DU, X., et al., "Influence of nano-silica agglomeration on fresh properties of cement pastes”, Construction and Building Materials, v. 43, pp. 557-562, Abril 2013.

[20] SENFF, L., HOTZA, D., REPETTE, W. L., et al., "Effect of nanosilica and microsilica on microstructure and hardened properties of cement pastes and mortars", Advances in applied ceramics, v. 109, n. 2, pp. 104-110, 2010.

[21] MAllmanN, P. F., Concreto de pós reativos: adição de nanosílica. Dissertação de M.Sc., Universidade Federal do Rio Grande do Sul, Porto Alegre, RS, Brasil, 2013.

[22] CASTRO, G. S., SOUZA, M. S. Estudo do índice de desempenho da nanossílica estabilizada em superplastificante adicionada a materiais cimentícios, Universidade de Brasília, Brasília, DF, 2016.

[23] NOLLI FILHO, P. R. Propriedades mecânicas do concreto de alto desempenho com adição de nanosílica estabilizada. Dissertação de M.Sc., Universidade Federal de Minas Gerais, Belo Horizonte, MG, Brasil, 2015.

[24] ASSOCIAÇÃO BRASILEIRA DE NORMAS TÉCNICAS, NBR 5733: Cimento Portland de alta resistência inicial, Jul. 1991.

[25] ASSOCIAÇÃO BRASILEIRA DE NORMAS TÉCNICAS, NBR NM 248: Agregados - Determinação da composição granulométrica, Jul. 2003.

[26] ASSOCIAÇÃO BRASILEIRA DE NORMAS TÉCNICAS, NBR NM 52: Agregado miúdo - Determinação da massa específica e massa específica aparente, Nov. 2009.

[27] ASSOCIAÇÃO BRASILEIRA DE NORMAS TÉCNICAS, NBR NM 45: Agregados - Determinação da massa unitária e do volume de vazios, Mar. 2006.

[28] ASSOCIAÇÃO BRASILEIRA DE NORMAS TÉCNICAS, NBR NM 30: Agregado miúdo - Determinação da absorção de água, Mai. 2001. 
[29] AMERICAN SOCIETY FOR TESTING AND MATERIALS, ASTM E1342-97: Standard Practice for Preservation by Freezing, Freeze-Drying, and Low Temperature Maintenance of Bacteria, Fungi, Protista, Viruses, Genetic Elements, and Animal and Plant Tissues, 2002.

[30] ASSOCIAÇÃO BRASILEIRA DE NORMAS TÉCNICAS, NBR NM 67: Concreto - Determinação da consistência pelo abatimento do tronco de cone, Mar. 1998.

[31] ASSOCIAÇÃO BRASILEIRA DE NORMAS TÉCNICAS, NBR 13278: Argamassa para assentamento e revestimento de paredes e tetos - Determinação da densidade de massa e do teor de ar incorporado, Out. 2005.

[32] ASSOCIAÇÃO BRASILEIRA DE NORMAS TÉCNICAS, NBR 7215: Cimento Portland - Determinação da Resistência a Compressão, Ag. 1996.

[33] ASSOCIAÇÃO BRASILEIRA DE NORMAS TÉCNICAS, NBR 7222: Concreto e Argamassa - Determinação da Resistência à tração por compressão diametral de corpos de prova cilíndricos, Jun. 2011.

[34] ASSOCIAÇÃO BRASILEIRA DE NORMAS TÉCNICAS, NBR 9778: Argamassas e concretos endurecidos - Determinação da absorção de água, índice de vazios e massa específica, Set. 2009. 